\title{
Spectrum Sensing Methodologies for Cognitive Radio Systems: A Review
}

\author{
Ireyuwa E. Igbinosa ${ }^{1}$, Olutayo O. Oyerinde ${ }^{2}$, Viranjay M. Srivastava ${ }^{1}$, Stanley Mneney ${ }^{1}$ \\ ${ }^{1}$ School of Electrical, Electronics and Computer Engineering \\ University of KwaZulu-Natal, Durban, 4041, South Africa \\ ${ }^{2}$ School of Electrical and Information Engineering, \\ University of the Witwatersrand, Johannesburg, \\ 2050, South Africa
}

\begin{abstract}
Spectrum sensing is an important functional unit of the cognitive radio networks. The spectrum sensing is one of the main challenges encountered by cognitive radio. This paper presents a survey of spectrum sensing techniques and they are studied from a cognitive radio perspective. The challenges that go with spectrum sensing are reviewed. Two sensing schemes, namely; cooperative sensing and eigenvalue-based sensing are studied. The various advantages and disadvantages are highlighted. Based on this study, the cooperative spectrum sensing is proposed for employment in spectrum sensing in wideband based cognitive radio systems.
\end{abstract}

Keywords-Cognitive radio; Cooperative sensing; Data Fusion; OFDM; Spectrum Sensing; wideband sensing

\section{INTRODUCTION}

Spectrum sensing can be said to be the process of performing measurement on a part of the spectrum and making a decision related to spectrum usage based upon measured data [1]. Spectrum sensing is a fundamental operational block of the cognitive radio (CR) which consists of spectrum sensing, management, sharing and spectrum mobility. The growing demand for wireless application has put a lot of strain on the usage of available spectrum. In order to address this situation and improve spectrum efficiency, Mitola proposed a technique that allows secondary users to utilize radio spectrum band allocated to primary users that is not actively used [2]. According to a report from the United States Federal Communication Commission [3], there are larger temporal and geographic variations in the utilization of allocated spectrum. It is also known that allocated spectrum is underutilized because of the static allocation of the spectrum. In order to overcome this, there is need to propose a means of improving utilization of spectrum $[4,5,6]$. The scarcity and underutilization of spectrum has led to the development of cognitive radio (CR) technology, which exploit the existing spectrum opportunistically. Cognitive radio technology was defined in [2] and [7]. In this paper, the definition of the FCC is adopted. It states "Cognitive Radio is a system that senses its operational electromagnetic environment and can dynamically and autonomously adjust its radio operating parameters to modify system operation, such as maximization of throughput, mitigation of interference and facilitation of inter-operability accessing the secondary markets', To achieve this goal of cognitive radio, it is a compulsory requirement that a cognitive user (CU) performs spectrum sensing to detect the presence of primary users' (PU) signal [8]. In the context of cognitive radio, the primary users can be defined as the users who have higher priority or right in the usage of a specific part of the spectrum. The secondary users (SU) on the other hand are the users who have lower priority or lower rights; they use the spectrum in such a way that they do not cause harmful interference to the primary users. However, secondary users need to have cognitive radio capabilities, such as sensing spectrum efficiently to ascertain if it is being occupied by a primary user and also change their radio parameters to exploit the unused part of the spectrum. The three popularly used methods for spectrum sensing are: Energy detection, Matched filtering and Cyclostationary detection $[9,10,11,12]$.The basic idea of cognitive radio is its ability for spectral reusing or spectrum sharing, which allows secondary users to communicate over licensed spectrum. It also involves determining what type of signals that are occupying the spectrum including modulation, waveform, bandwidth, carrier frequency, etc. This however, requires powerful signal analysis method with additional computational complexity. Wideband spectrum sensing for cognitive radio network has not been sufficiently investigated in literature. Earlier approach uses a tunable narrowband filter and the RF front-end to sense one narrow frequency band at a time [13], in which the existing narrowband sensing techniques can be applied. In order to operate over multiple frequency bands at a time, the RF front-end needs wideband architecture. Spectrum sensing usually involves the estimation of the power spectral density (PSD) [14, 15]. There are so many factors that can cause spectrum sensing to be practically challenging. The rest of the paper is organized as follows. Complexity of spectrum sensing concept is studied in Section II. The challenges associated with spectrum sensing for cognitive radio are discussed in section III. Section IV shows the algorithms for spectrum sensing in cognitive radio. Section $\mathrm{V}$ discusses the cooperative spectrum sensing. Section VI discusses the research challenges involved in improving cooperative spectrum sensing and finally section VII concludes this paper. 


\section{COMPLEXITY OF SPECTRUM SENSING CONCEPT}

Spectrum opportunity is conventionally defined in literature as "a band of frequencies that are not used by a primary user of that band at a particular time and specific geographic location," [16]. This definition therefore introduces multi-dimensional spectrum awareness, since a spectrum hole is a function of frequency, time and geolocation. Since noise is present all the time in the entire radio spectrum, then an empty frequency bin doesn't exist. [17] Therefore it is important to be able to differentiate a band occupied by a primary user signal (PU) and the one from a spectrum hole that contains noise only signal. The traditional definition of spectrum sensing only exploits the three dimensions of the spectrum space. These are frequency, time and geo-location. Traditional methods usually relate to sensing the spectrum using these three [18]. However there are other dimensions that can be exploited for further spectrum opportunity. For example the code dimension of the spectrum space has not been extensively explored in details in literature therefore the traditional spectrum sensing algorithms find it challenging to deal with signals that makes use of spread spectrum, time or frequency hopping codes. As a result, this type of signals causes a lot of challenges in spectrum sensing as discussed in the later part of this paper. Also the angle dimension is another area which is coming up as there are recent advances in multi- antenna technologies such as beam forming, multiple users can be multiplexed into the same channel at the same time in the same geo-location. Hence, in angle dimension, a primary user and a secondary user can be in the same geo-location and share the same channel. Spectrum sensing should include the process of recognizing occupancy in all dimensions of spectrum space and find spectrum holes. For instance, a certain frequency can be occupied for a given time, but may be empty in another time. Hence, a temporal dimension is as important as frequency dimension. The idle periods between bursty transmissions of local area network (WLAN) signals are exploited for opportunistic usage [19]. As a result of this requirement, advanced spectrum sensing algorithms that offers spectrum awareness in multiple dimensions can be developed.

TABLE I. SUMMARY OF DIMENSIONAL SPECTRUM AWARENESS

\begin{tabular}{|c|c|c|}
\hline Dimensions & Sensing Parameters & Observations \\
\hline Frequency & $\begin{array}{l}\text { Frequency domain } \\
\text { opportunity }\end{array}$ & $\begin{array}{l}\text { Spectrum opportunity in this dimension means that all bands are not used } \\
\text { simultaneously at the same time (some bands may be available for opportunistic } \\
\text { usage). }\end{array}$ \\
\hline Time & $\begin{array}{l}\text { Opportunity of } \\
\text { Specific band in time }\end{array}$ & $\begin{array}{l}\text { This involves the availability of a specific part of the spectrum in time. In } \\
\text { other words, the band is not continuously used. Hence there would be times where } \\
\text { it would be available for opportunistic usage. }\end{array}$ \\
\hline $\begin{array}{l}\text { Geo- } \\
\text { Location }\end{array}$ & $\begin{array}{l}\text { Location and distance } \\
\text { of primary users }\end{array}$ & $\begin{array}{l}\text { The spectrum can be available in some parts of the geo-location and occupied } \\
\text { in some other part at a given time. This takes advantage of pathloss in space. This } \\
\text { measurement can be avoided by simply looking at the interference level. However, } \\
\text { one needs to be careful of hidden terminal problem. }\end{array}$ \\
\hline Code & $\begin{array}{l}\text { Time hopping (TH) or } \\
\text { frequency hopping (FH) } \\
\text { sequences used by the } \\
\text { primary users. }\end{array}$ & $\begin{array}{l}\text { The spectrum over a wideband can be used at a given time through spread } \\
\text { spectrum or frequency hopping. This doesn't mean that there is no availability over } \\
\text { this band. Hence, simultaneous transmission without interfering with primary } \\
\text { users would be possible in code dimension with an orthogonal code with respect to } \\
\text { codes that primary users are using. (Not only detecting the usage of the spectrum, } \\
\text { but also determining the used codes, and possibly multipath parameters as well). }\end{array}$ \\
\hline Angle & $\begin{array}{l}\text { Beam (azimuth, } \\
\text { elevation angle) and } \\
\text { locations of primary users }\end{array}$ & $\begin{array}{l}\text { Along with the knowledge of the location/position or direction of primary } \\
\text { users, spectrum opportunity in angle dimension can be created. For instance, if a } \\
\text { primary user is transmitting in a specific direction, the secondary user can transmit } \\
\text { in other directions without causing interference on the primary user. }\end{array}$ \\
\hline
\end{tabular}

\section{THE CHALLENGES ASSOCIATED WITH SPECTRUM SENSING FOR COGNITIVE RADIO}

Before getting into the details of spectrum sensing techniques, it is advisable to review the challenges. The challenges associated with the spectrum sensing for cognitive radio are discussed in this section.

\section{A. Hardware Requirements}

Applications for spectrum sensing in cognitive radio needs high sampling rate, high resolution analog to digital converter (ADC) with large dynamic range and high speed signal processors. Noise variance estimation technique has been popularly used for optimal receiver [14]. Designs such as, channel estimation, soft information etc., as well as improved handoff, power control and channel allocation techniques [20]. The noise and estimation challenges are easier for these purposes as receivers are tuned to receive signals that are transmitted over a desired bandwidth. However, receivers have the ability to process the narrowband baseband signals with reasonably low complexity and low power processors. Cognitive radio terminals are then required to process transmission over a wideband for utilizing any opportunity [18]. Hence, a cognitive radio should be able to capture and analyze a relatively larger band and utilize any spectrum opportunities. These large operating bandwidths create additional requirements on the radio frequencies (RF) components such as antennas and power amplifiers. Hence high speed processing units are needed for performing computationally demanding signal processing task with 
relatively low delay. Spectrum sensing can be performed through two different architectures, such as single radio and dual radio. In the case of the single radio, only one specific time slot is assigned for spectrum sensing. Due to the limitation in sensing time, only certain accuracy can be guaranteed for spectrum sensing result. However, the spectrum sensing efficiency is decreased as some part of the available time slot is used for sensing instead of data transmission. The merit of using the single radio architecture is because of its simplicity and low cost of implementation. However, in the dual radio architecture, one radio chain is allocated for data transmission and reception while the other chain is allocated for spectrum monitoring [21]. The limitations of such approach is that it increases power consumption and hardware cost. In practice, there are already available hardware and software platforms for cognitive radio such as GNU Radio, Universal Software Radio Peripheral and shared spectrum's XG Radio. Energy detector based sensing is mainly used in this platform because of its simplicity.

\section{B. Hidden Primary User Problem}

The hidden primary user problem is caused by several factors such as: - severe multipath fading or shadowing observed by secondary users while scanning for primary users. In this condition, the cognitive radio devices causes, unwanted interference to the primary user (receiver) as the primary transmitter, signal cannot be detected due to the location of devices. Cooperative sensing has been proposed as a means of handling hidden primary user problem [22, 11, 23]. Cooperative sensing is discussed in detailed in the later part of this paper.

\section{Detecting Spread Spectrum Primary Users}

There are two main types of technologies for detecting commercially available devices, they are: fixed frequency and spread spectrum. There are two main spread spectrum technologies available. The frequency hopping spread spectrum (FHSS) and direct sequence spread spectrum (DSSS). Fixed frequency devices operate at a single frequency or channel [18]. An example of this kind of system is the IEEE $802.11 \mathrm{a} / \mathrm{g}$ based WLAN. FHSS devices have the ability to change their operational frequencies dynamically into multiple narrowband channels. This technique is known as hopping and is implemented according to a sequence that is known to both transmitter and receiver. However, they use a single band to spread their energy. Primary users that use spread spectrum signaling are difficult to detect as the power of the primary user is distributed over a wide frequency range even though the actual information bandwidth is much narrower [11]. This problem can however be avoided partially if the hopping pattern is known and perfect synchronization to the signal can be achieved.

\section{Sensing Duration and Frequency}

In order to prevent interference to and from primary license owner, cognitive radio should be able to identify the presence of primary users as quickly as possible and should exit the band immediately. Therefore, sensing methods should be able to identify the presence of primary users within certain duration. This requirement poses a limit on the performance of sensing algorithms and creates challenges for cognitive radio design. Selection of sensing parameters brings about tradeoff between the speeds (sensing time) and reliability of sensing. Sensing frequency is a design parameter that needs to be chosen carefully [23]. In a case when the status of primary users is known to change slowly, sensing frequency requirements can be relaxed. In addition to sensing frequency, the channel detection time, channel move time and some other timing related parameters are also defined in [24]. Another factor that can affect the sensing frequency is the interference tolerance of primary license owners. An example is when a cognitive radio is using opportunities in public safety bands, sensing should be done as frequently as possible in order to prevent any interference. The effect of sensing time on the performance of secondary users is investigated in [25].The aim is to maximize the average throughput of secondary users while guiding primary users from interference [18]. Similarly, detection time is obtained using numerical optimization in [26]. Channel efficiency is maximized for a given detection probability. Sensing time can be decreased by sensing only changing parts of the spectrum instead of the entire target. A channel that is being used by secondary users cannot be used for sensing. Hence, secondary users have to stop data transmission for spectrum sensing [27]. This however, decreases the spectrum efficiency of the overall system [23]. To solve this problem, a method known as dynamic frequency hopping (DFH) is proposed. The DFH method is based on the assumption of having more than a single channel. This was proposed in [28].

\section{E. Decision Fusion in Cooperative Sensing}

Sharing information among cognitive radios and combining results from various measurements is a challenging task [18]. This shared information can either be soft or hard decisions made by each cognitive device [29]. The results presented in [29, 30] shows that soft information combining method performs better than hard information combining method in terms of the probability of missed opportunity. Hard decision is found to perform as good as soft decision when the numbers of cooperating users are high. The optimum fusion rule for combining sensing information is the ChairVarshney rule with log-likelihood ratio test [31]. Likelihood ratio tests (LRT) are used for making classification using decision from secondary users in [29, 32, 33, 34, 35]. Different techniques for combining sensing result are employed in [12]. The credibility of cognitive radios depends on the channel conditions and their distance from a licensed user. The required number of nodes for satisfying a probability of false alarm rate is investigated in [36].

\section{F. Security}

In Cognitive radio, an unauthorized user can change its air interface to look like a primary user. However, this phenomenon misleads the spectrum sensing performed by legitimate primary users. This type of attack is investigated in [37]. The possibilities of primary user emulation (PUE) attacks are realistic due to the facts that $\mathrm{CR}$ is highly reconfigurable due to the fact that they are software based air interface [16, 38]. In order to stop such attacks, a robust transmitter verification scheme that can distinguish between legitimate incumbent primary signal transmitters and secondary signal transmitters needs to be designed [16]. The 
task of differentiating an incumbent primary signals user from secondary users becomes challenging when the requirement described in FCC's NPRM 03-322, which states that " no modification to the incumbent system should be required to accommodate opportunistic use of the spectrum by secondary user signal”. The major technical challenge in spectrum sensing is distinguishing primary signals from secondary user signal. A public key encryption is proposed in [39]. The primary user encrypts its identification with its private key and appends the encrypted value (signature) to its transmission. All secondary users scan for the signature, during the sensing period, the signature from various base stations. The base station then verifies these signatures. Since the primary user knows its signature, a malicious secondary user cannot produce a valid signature.

\section{AlgORITHM FOR SPECTRUM SENSING IN COGNITIVE RADIO}

This section presents a study on spectrum sensing techniques that require knowledge of both source signal and noise power information. Some of the most common spectrum sensing techniques in this category is explained in this section.

\section{A. Parametric Method of Spectrum sensing schemes}

Three basic parametric method of spectrum sensing are explained as follows:

\section{a) Optimal LRT-Based Sensing}

The Neyman-Pearson states that for a given probability of false alarm, the test statistics that maximizes the probability of detection is the likelihood ratio test (LRT) [40, 41, 42] which is defined as:

$$
T_{L R T}(x)=\frac{P\left(x \mid H_{1}\right)}{p\left(x \mid H_{0}\right)}
$$

where $\mathrm{P}$ (.) denotes the probability density function (PDF) and $(\mathbf{x})$ denotes the received signal vector that is the aggregation of $\mathrm{x}(\mathrm{n}), \mathrm{n}=0.1 \ldots \ldots, \mathrm{N}-1$. Such likelihood ratio test decides $\mathcal{H}_{1}$ when $T_{\text {LRT }}(x)$ exceeds a threshold $\gamma$, otherwise it uses $\mathcal{H}_{0}$. The main challenge in in implementing the LRT is the requirement on the distribution give in equation (1). The distribution of random vector x less than $\mathcal{H}_{1}$ is related to the source signal distribution, the wireless channels and the noise distribution. The distribution of $\mathrm{x}$ under $\mathcal{H}_{0}$ is related to the noise distribution [43].

In order to implement the LRT, a prior knowledge of the channel as a well as the signal and noise distribution is of paramount importance. This is practically difficult to realize.

Assuming that the channels are flat-fading and the received source signal sample $s_{i}(n)$ is independent over, the PDF in LRT is decoupled as:-

$$
P\left(x \mid H_{1}\right)=\prod_{n=0}^{N-1} P\left(x(n) \mid H_{1}\right),
$$

$$
P\left(x \mid H_{0}\right)=\prod_{n=0}^{N-1} P\left(x(n) \mid H_{0}\right)
$$

Furthermore assuming that noise and signal samples are both Gaussian distributed, such that $\eta(\mathrm{n}) \sim \mathrm{N}(0, \sigma 2 \eta I)$ and $\mathrm{s}(\mathrm{n}) \sim \mathrm{N}(0, \mathrm{Rs})$, the LRT becomes the estimator correlator (EC) detector as shown in [54] for which test statistic is given as:

$$
T_{E C(x)}=\sum_{n=0}^{N-1} x^{T}(n) R_{s}\left(R_{s}+{ }^{\sigma^{2}} 1\right)^{-1} x(n)
$$

From equation (3) it is shown that $\mathrm{R}_{\mathrm{s}}\left(\mathrm{R}_{\mathrm{s}}+2 \sigma^{2} \eta \mathrm{I}\right)^{-1} \mathrm{x}(\mathrm{n})$ is the minimum mean squared error (MMSE) estimation of the source signal $s(n)$. Thus, $T_{E C}(x)$ in (3) can be seen as the correlation of the signal $\mathrm{x}(\mathrm{n})$ with the MMSE estimation of $\mathrm{s}$ (n). EC detector needs to know the source signal covariance matrix $\mathrm{R}_{\mathrm{s}}$ and noise power $\sigma^{2} \eta$. Hence when the signal presence is unknown it becomes unrealistic to require signal covariance matrix for detection. It should be noted that if we assume that the noise is Gaussian distributed and the signals source is deterministic and known to the receiver, which in this case is the radar signal processing [44, 45, 46], it would then it can be easy to show that LRT becomes the matched filter based detector and its test statics is [43].

\section{b) Matched Filter}

Matched filter (MF) is a linear filter designed to maximize the output signal to noise ratio (SNR) for a given input signal [47]. Matched filtering is also known as optimal method for detection of primary users when transmitted signal is known [48]. Hence, cognitive radio has a prior knowledge of the Primary User Signal at both PHY and MAC layer, such as bandwidth, frequency, modulation type to demodulate received signals [49]. Matched filter detector has a high processing gain, but the sensing devices have to achieve coherency and demodulate primary user signal. This can be achieved since most wireless networks have pilot patterns (or symbols) and preambles that can be used for coherent detection. For examples: TV Signal has narrowband pilot for audio and video carriers; CDMA system have dedicated spreading codes for pilot and packet acquisition. The operation of matched filter detection is expressed as:

$$
Y[n]=\sum_{K=-\infty}^{\infty} h[n-k] x[k]
$$

where $\mathrm{x}$ is the unknown signal (vector) and is convolved with the h. The impulse response of the matched filter is useful only in cases where the information from the primary users is known to the cognitive users.

Matched filter advantage is it requires less detection time because it requires only $\mathrm{O}(1 / \mathrm{SNR})$ samples to meet a given probability of detection constraint. When the information of the primary user is known to the cognitive radio user, matched detection is optimal [64]. 
The drawback of matched filter is that it requires prior knowledge of every primary signal. If the information is not accurate, MF would perform poorly. Also the most significant disadvantage of $\mathrm{MF}$ is that cognitive radio would need a dedicate receiver for every type of primary user [61].

\section{c) Cyclostationary Based Detection}

Cyclostationary based detection is a method that detects primary users by exploiting its Cyclostationary features of the received signals $[50,51]$. Modulated signals are in general coupled with sine wave carriers, pulse trains, repeating spreading, hoping sequence or cyclic prefixes; these modulated signals are known as cyclostationary, since they have statistics, mean and autocorrelation. They can also be intentionally induced to assist spectrum sensing [52]. The cyclostationary based detection algorithm can differentiate noise from primary users signal. This is due to the fact that noise is in wider sense stationary with no correlation while modulated signal are cyclostationary with spectral correlation due to the redundancy of signal periodicities [43]. This periodicity trend is used for analyzing various signal processing tasks such as detection, recognition and estimation of the received signals. Even though cyclostationary feature detection have high computational complexity, it performs well satisfyingly well under low SNR due to its robust against unknown level of noise. Free bands in the spectrum are detected following the hypotheses testing problem in received signal $x(t)[53]$.

$$
x(t)=s(t) h+w(t)
$$

where $\mathrm{s}(\mathrm{t})$ is the modulated signal, $\mathrm{h}$ is channel coefficient and $\mathrm{w}(\mathrm{t})$ AWGN.

- Under $H_{0} x(t)$ it is not cyclostationary and thus the band is considered free

- Under $H_{1} \quad x(t)$ is cyclostationary and thus the band is considered congested

where $H_{0}$ signifies the existence of signals and $H_{1}$ the existence of signal. Modulated signal $x(t)$ is considered to be a periodic signal or a cyclostationary signal in wide sense its mean and autocorrelation exhibits periodicity as shown in [54].Though cyclostationary detection has certain advantages such as its robustness to uncertainty in noise power and propagation channel. It has its own disadvantages as follows:

- It needs a very high sampling rate

- The computation of spectral correlation density (SCD) function would require large number of samples and thus become complex.

- The strength of SCD could be affected by the unknown channel

- Sampling time error and frequency offset could affect the cyclic frequency.

\section{B. Semi Blind Detection Methods}

This section shows detection techniques that requires only noise power information. Hence it's called semi-blind detection.

\section{a) Energy Detection}

Energy detection is an optimal way to detect primary signals when prior information of the primary signal is unknown to secondary users. It measures the energy of the received waveform over a specified observation time [9, 55]. In addition, as receivers do not require any knowledge on the primary users signal. The signal is detected by comparing the output of the energy detector with a threshold which depends on the noise floor. Energy detector also known as radiometer has been investigated and widely used for signal detection due to its advantage of simple circuitry in practical implementation [56]. Prior to energy detection been proposed, many work have been performed to study energy detection based schemes in radar and security communication areas. Have some advantages that motivate research in this area. These include the following:-

- It is more generic as receivers do not need any knowledge on primary user's signal

- It is very simple to implement

The signals can be detected at low SNR provided the detection interval is adequately long and noise power spectral density is known.

The study of energy detection takes into account the dynamics traffic patterns of primary users, in the form random signal arrival and departure is of theoretical and practical importance. However, some of the existing techniques resort to approximation to certain approximation techniques to characterize the detection performance. In order to improve this technique, we would propose a Bayesian based Energy detection algorithm. There has been recent works which addresses the effect of primary user traffic patterns on the performance of the detection of energy detectors. In [57], they considered the random arrival or departure of the primary user's signal which exploits the distributions of the arrival and departure times. The effect of the primary user traffic on the detection performance is investigated and studied in [58]. However, to improve the robustness of energy detection we would propose a Bayesian -based Energy detection by exploiting the statistical knowledge.

\section{b) Wave form based Sensing}

In wireless systems, known patterns such as preambles, midambles, regularly transmitted pilot pattern, spreading sequence etc. [56]. The problems of energy detection which are false detection and difficulty in differentiating modulated signals from interference. Both of these problems are addressed in waveform based sensing. Waveform based sensing is performed in time domain using received signal;

$$
y(n)=x(n)+z(n)
$$

Where $x(n)$ is the signal to be detected and $z(n)$ is the Additive white Gaussian noise (AWGN). Assuming the known time- domain signal contains $\mathrm{N}_{\mathrm{B}}$ signal [56]. We can then consider the following wave forms sensing metric:

$$
S=R_{e}\left[\sum_{n=1}^{N_{B}} y(n) x^{*}(n)\right]
$$


When there is no primary user signal present, the sensing metric would then be

$$
S=S_{0}=R_{e}\left[\sum_{n=1}^{N_{B}} z(n) x^{*}(n)\right]
$$

When there is presence of primary user's signal present the sensing metric becomes:

$$
S=S_{1}=\sum_{n=1}^{N_{B}}|x(n)|^{2}+R_{e}\left[\sum_{n=1}^{N_{B}} z(n) x^{*}(n)\right]
$$

The decision on the presence of a primary user can be made by comparing the decision metric $S$ against a fixed threshold $\lambda_{z}$. The sensing metrics (7) can then be approximated as a Gaussian random variable when $N_{B}$ sample is large. [59].Waveform based sensing outperforms energy detection based sensing in reliability and convergence time. Though waveform based sensing has good advantage, it also has its drawback. Since waveform based sensing requires short measurement time, it is then susceptible to synchronization errors.

\section{Totally Blind Detection}

This section presents detection techniques of spectrum sensing that requires no information what so ever on source signal or power. These techniques are explained as follow:

\section{a) Eigenvalue based-sensing}

This section reviews two sensing algorithms under the totally blind sensing spectrum. The first algorithm is based on the ratio of the maximum eigenvalue to minimum eigenvalue and the other is based on the ratio of average eigenvalue to minimum eigenvalue. There are two major eigenvalue based detection technique that would be studied in this paper, they are:

\section{1) Maximum-minimum eigenvalue detection (MME)}

This method generalizes the energy detection because it is used on a basis similar to the energy detection. What makes this unique is that it does not require any prior knowledge of the signal and the channel. It also eliminates the susceptibility of energy detection synchronization error, since it doesn't require synchronization. It is shown that the ratio of the maximum eigenvalue to the minimum can be used to detect signal [59]. This is achieved by some Random matrix theories (RMT), from this we can quantize the ratio and therefore find the threshold. The probability of the false alarm can also be found by using the random matrix theories $[60,4]$. This technique overcomes the noise uncertainty difficulty which is peculiar to the energy detection while keeping the advantages of energy detection. It can even perform better than energy detection when the signals to be detected are highly correlated for signal detection as we already know from the beginning of this paper, there are two hypotheses $H_{0}$, signal does not exist and $H_{1}$ signal exist. The received signal under the hypothesis is given as follows $[13,40]$ :-

$$
H_{0}: x(n)=\eta(n)
$$

$$
H_{1}: x(n)=s(n)+\eta(n),
$$

where $s(n)$ is the transmitted signal sample and $\eta(n)$ is the white noise which is independent and identically distributed (iid). There are two probabilities that are of interest for channel sensing. They are; probability of $\operatorname{detection} P_{d}$, at hypothesis $H_{1}$ and the probability of the sensing algorithm having detected the presence of primary signal. The probability of false alarm $P_{F A}$. which defines the Hypothesis $H_{1}$. [60]. The probability of the presence of the primary signal can be defined by the following vectors. Assuming we consider $\mathrm{L}$ consecutives samples and then defines the vectors as follows [61]. The major advantage of the maximumminimum eigenvalues based detection is that they do need the noise power for detection. The major similarity with the energy detector is that they both use the received signal for detection and no information on the transmitted signal and channel is needed.

\section{2) Energy with Minimum Eigenvalue based Detection} (EME)

In this algorithm, the ratio of the signal energy to the minimum eigenvalue is used for detection of the primary user signal. as discussed in [62]. The diffrence between the conventional energy detection and EME is:

- Energy detection compares the signal energy to the noise power, which has to be estimated in adavance.

While the EME on the other hand compares the signal energy to the minimum eigenvalues of the sample covariance matrix, which is computed from the received signal only. Though they have differences, but are however similar to energy detection. The MME and EME only use the received signal samples for detection and requires no information on the transmitted signal and channel is needed. The major advantage of EME detection over energy detection is:

- Energy detection requires noise power for detection while the EME does not.

The major complexity of EME is the computation of the covariance matrix equations and the eigenvalue decomposition of the covariance matrix. From the work done by Zeng et al. [62], the EME is worse than the ideal energy detection but better than energy detection with noise uncertainty $0.5 \mathrm{~dB}$ [62]. The MME on the other hand performs better than the EME from the experiment done by Zeng et al [60]but there is no theoretical proof yet in literature yet. The eigenvalue based methods can be used for different signal detection application without the knowledge of the signal, channel and noise power such and DTV signal and wireless microphone.

\section{COOPERATIVE SPECTRUM SENSING}

This scheme was proposed as a solution to the problem of noise uncertainty, fading and shadowing. This scheme decreases the probabilities of false detection and false alarm. Cooperative sensing can also be used to solve the problem of hidden primary user problem and can also reduce sensing time [18]. The major idea of cooperative sensing is that it increases the sensing performance by exploiting the spatial diversity in the observation of spatially located cognitive radio users [19]. 
By cooperating, cognitive radio users can share their sensing information for making a combined decision more accurate than individual decision [51]. The process of sensing starts with local sensing; this is when spectrum sensing is performed individually at each cognitive radio. The local sensing can be formulated as hypothesis problem as follows [63].

$$
x(t)= \begin{cases}n(t) & H_{0} \\ h(t), s(t)+n(t), & H_{1}\end{cases}
$$

Where $x(t)$ is the received signal at the cognitive radio user, $s(t)$ is the transmitted primary signal, $n(t)$ is the zero mean additive white Gaussian noise (AWGN), $H_{0}$ and $H_{1}$ denote the hypothesis of the absence and presence of signals respectively. The detection performance probability and the probability of false alarm are defined as:

$$
\begin{gathered}
P_{d}=P\left\{\text { decision }=H_{1} / H_{1}\right\}=P\left\{\Upsilon>\lambda \mid H_{1}\right\} \\
P_{f}=P\left\{\text { decision }=H_{1} / H_{0}\right\}=P\left\{\Upsilon>\lambda \mid H_{0}\right\},
\end{gathered}
$$

Where $\Upsilon$ is the decision statistics and is $\lambda$ the decision threshold.

Cooperative spectrum sensing implementation can be categorized into three, they include: Centralized, Distributed and Relay assisted.

\section{a) Centralized Cooperative Sensing}

In the centralized cooperative sensing, the central identity also known as fusion center (FC) [62] controls the three steps of cooperative sensing process. The first stage of the process, FC then chooses a channel or frequency band for sensing then delegates all cooperating cognitive radio to individually perform local sensing [64]. In the second process, all cooperating cognitive radio reports the sensing results through the control channel. In the third process the FC combines the received local sensing information, then determines the presence of primary users and then passes the decision to the cooperating cognitive radio users.

All cognitive radio users are tuned to the selected channel or frequency band where a wireless point to point link between the primary user (PU) transmitter and each cooperating radio also known as sensing channel is used for observing the PU and for data reporting all cognitive users are tuned to a control called a reporting channel. From figure 1, the Fusion center (FC) and CR1-CR5 performing local sensing and reporting back to the FC. In centralized system, the cognitive radio base station is the FC. But in cognitive Radio ad hoc network (CRAHNs), the cognitive radio base station is not present; hence any cognitive radio can then act as a FC to coordinate the sensing activities and then combines the sensing information from the cooperating neighbors. In a situation where there are large numbers, the required bandwidth for reporting results becomes huge. The reduction techniques of the sharing bandwidth and local observation are discussed in [65]. Only cognitive radios with reliable information are required to send decision to the fusion center [66].
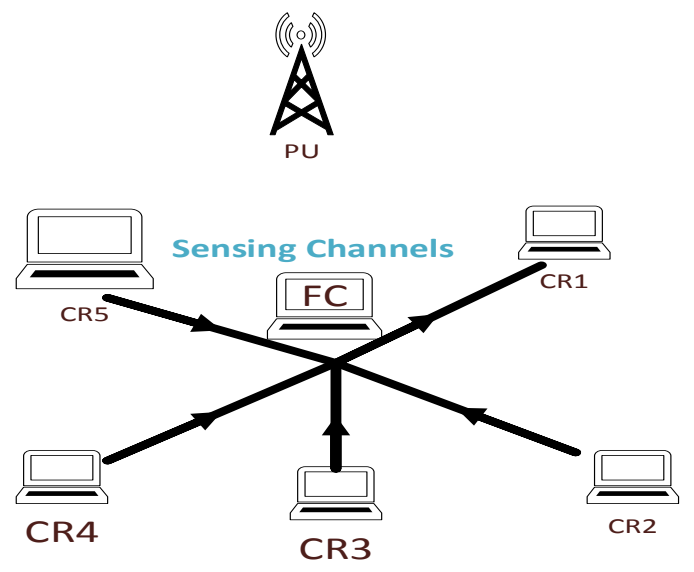

Reporting Channels

Fig. 1. Illustration of Centralized Cooperative sensing

\section{b) Distributed Cooperative Sensing}

In this type of sensing, cognitive node share information among each other. Though, they make their individual decision on the presence or absence of primary users. Fig 3 shows the distributed cooperative sensing. CR1-CR5 shares the locally sensed results with users within the transmission range. Several distribution algorithm have been developed [66, 17, 67], based on these algorithm each cognitive radio user transmits its own data to other users, then combines its results with the received data and then decides if a primary user is present by using local criterion [64],Distributed sensing is more advantageous than the centralized sensing because it does not require fusion center (FC) for cooperative decision thereby reducing cost.

\section{c) Relay Assisted Spectrum Sensing}

Since the centralized and distributed cooperative sensing scheme is not that perfect, it gave birth to the relay assisted scheme. In this scheme, the cognitive radio user observing a weak sensing channel and a strong report channel, a cognitive radio user with a strong sensing channel and a weak report channel [64].

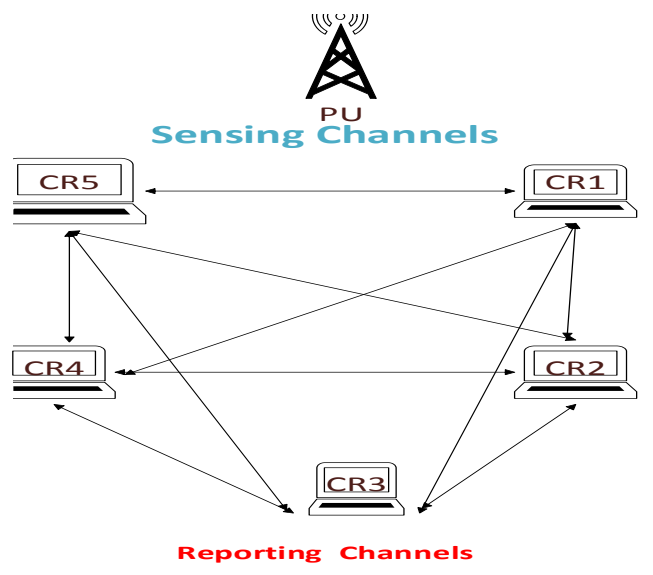

Fig. 2. Illustration of Distributed Cooperative sensing 
Figure 2, shows CR1, CR4 and CR5, observes strong primary user signal, which may suffer from a weak reporting channel. CR2 and CR3 which have a strong report channel, serves as a relay to the fusion center (FC). In this situation, the report channels from CR2 and CR3 can also be known as the relay channels. Though, figure 2 shows a centralized structure. The relay assisted cooperative sensing scheme can also exist in distributed scheme. Hence, if the centralized and distributed structures are one hop cooperative sensing, the relay assisted structure can be considered as multi -hop cooperative sensing [17]. Though, the cooperative sensing scheme has some impressive advantages, such as higher accuracy in primary user detection, reduced sensing time and the prevention of shadowing effect and hidden node problem. The disadvantage with the scheme is the complexity of sensor within the cooperation among system cooperation, traffic overhead and the need for a control channel.

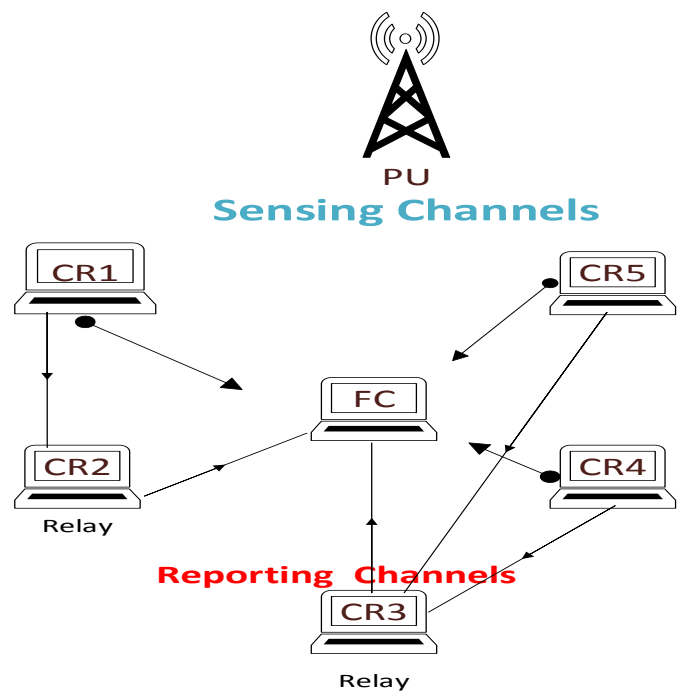

Fig. 3. Illustration of Relay Assisted Cooperative sensing

\section{d) Data Fusion}

In cooperative spectrum sensing data fusion is a procedure of combing local sensing data for hypothesis testing that is a constituent of cooperative sensing. This is based on the control channel bandwidth requirement; recorded sensing results can be of different forms, types and sizes [19]. Hence, the sensing results relayed to the FC or shared with cooperating users can be combined in three different ways they include; soft combing, quantized soft local combining and hard local decision

In the case of soft combining, cognitive radio users can either transmit the whole local sensing samples or the total local test statistics for soft decision. The receiver diversity techniques that is utilized for soft combining is the equal gain combining (EGC) and maximal ratio combining (MRC) [68]. The cognitive users can only transmit the quantize local sensing results and send the quantized data for soft combining increase control communication cost. In the case of hard combining, the commonly used fusion rules are AND, OR and Majority Rules. The cognitive radio users make a local decision and transmit the binary decision for hard combing.

\section{RESEARCH CHALLENGES TO IMPROVE EXISTING COOPERATIVE SENSING}

The challenges to improve cooperative sensing delay are as follows:

Multiple tradeoffs in cooperative sensing delay: The sensing-throughput tradeoff analysis in cooperative Sensing should consider not only the sensing time and CR throughput, but also the report delay and the delay for synchronization or asynchronous reporting. Thus, the challenge is to balance the tradeoff between the CR throughput and cooperative sensing delay, which consists of multiple delay components depending on the cooperative sensing schemes.

Delay analysis in distributed schemes: Distributed cooperative sensing schemes usually require an iterative process to reach the cooperative decision. The cooperative sensing delay is dominated by the report delays if the number of iterations for convergence is large. As a result, the delay analysis and the convergence of the distributed cooperative algorithm should be jointly considered.

With the above listed factors, we would improve the cooperative spectrum sensing by using an improved energy detection based on second order statistics in a centralized cooperative spectrum sensing scheme.

\section{CONCLUSION}

In this paper, the various spectrum sensing schemes have been reviewed. The various aspects of the sensing scheme are explained in details. Based on the different methodologies that were studied, the cooperative sensing scheme was considered as a solution to some specific challenges associated with spectrum sensing such as hidden primary user etc. Cooperative sensing is seen as an effective technique to improve detection performance by exploiting spatial diversity. Special attention was also given to the totally blind sensing methods that do not require prior information on the source signals and the transmitting channel. In conclusion, the review of various sensing techniques would be useful to researchers in developing a novel system for spectrum sensing algorithm. Also we identified some challenges in cooperative spectrum sensing which would be useful to researchers starting their research.

\section{REFERENCES}

[1] " End to End Efficiency (E3)," 2009. [Online]. Available: http://icte3.eu.

[2] J.Mitola, "Cognitive Radio: Making software radio more personal," IEEE personal communication, vol. 6, no. 4, pp. 13 - 18, Aug 1999.

[3] FCC, "Spectrum policy task force report,," Technical report 02-135 Federal communication commission, Nov 2005.

[4] A.M.Shahzad, M.A.Shah, A.H.Dar,A.Haq, A.U.Khan, T.Javed, S.A.Khan, "Comparative analysis of primaty transmitter detection based spectrum sensing technologies in cognitive radio systems," Australian Journal of Basic and applied sciences, vol. 4, no. 9, pp. 4522 - 4532, 2010.

[5] W.Wang, "Spectrum sensing for cognitive radio," third international symposium on intelligent information technology application workshop, pp. 410 - 412, 2009.

[6] V.Stoianovici, V.Popescu, M.Murroni, "A Survey on spectrum sensing techniques in cognitive Radio.," Bulletin of the Transilvania University of Brasov, vol. 15, no. 50. 
[7] J.Mitola, "Cognitive radio: An Integrated agent architecture for software definded radio," PHD thesis, Royal Institute of technology (KTH), May 2000.

[8] C.H.Hwang, S.H.Chen, "Spectrum sensing in wideband OFDM cognitive radio," IEEE transaction in signal processing, vol. 58, no. 2, pp. 709 - 719, Feb 2010.

[9] H.Urkowitz, "Energy detection of unknown deterministic signals," Proceeding of the IEEE, vol. 55, no. 4, pp. 523 - 531, 1967.

[10] M.Ghozzi, M.Doiler, F.Marx and J.Palicot, "Cognitive radio:Methods for detection of free bands," Comptes Rendus Physique,Elservier, vol. 7, pp. 794 - $804,2006$.

[11] D.Cabric, A.Tkachenko and R.Brodersen, "Spectrum sensing measurement of pilot, energy and collaborative detection," in IEEE military communication conference, washington DC, USA, 2006.

[12] F.F.Digham, M.S.Alouini and M.K.Simon, "On Energy detection of unknown signals over fading channels," vol. 55, no. 1, pp. 21 - 24, 2007.

[13] A.Sahai and D.Cabric, "Spectrum sensing; Fundamental limit and practical challenges," in IEEE international symposium on new frontiers in dynamic spectrum access network (DySPAN '05), Baltimore, MD, USA, 2005.

[14] H.Sun and A.Nallanathan, "Wideband spectrum sensing for cognitive radio network: A Survey," IEEE wireless communication, vol. 20, no. 2, pp. 74-81, April 2013.

[15] H.Sun, W.Y.Chiu,J.Jiang, A.Nallanathan and H.V.Poor, "Wideband spectrum sensing with sub-Nyquist samples in cognitive radios," IEEE Transaction on signal processing, vol. 60, no. 11, pp. 6068 - 6073, 2012.

[16] S.Haykin, "Cognitive radio: Brain-Empowered wireless communication," IEEE journal on selected areas in communication, vol. 25, pp. 201 - 220, Feb 2005.

[17] J.A.Bazerque and G.B.Giannakis, "Distributed spectrum sensing for cognitive radio networks by exploiting sparsity," IEEE transaction on signal processing , vol. 58, no. 1, pp. 1847 - 1862, Mar 2010.

[18] T.Yucek and H. Arslan, "A survey of spectrum sensing algorithms for cognitive radio applications," IEEE communications and survey\& tutorial , vol. 11, no. 1, 2009.

[19] I.F.Akyildiz, F.Brandon, L.Ravikumar, "Cooperative spectrum sensing in cognitive radio networks:A survey," Physical communication, vol. 4, pp. 40 - 62, 2011.

[20] T.Yucek and H.Arslan, "MMSE Noise plus interference power estimation in adaptive OFDM system," IEEE transaction on vehicular technology, vol. 56, no. 6, pp. 3857 - 3863, Nov 2007.

[21] Y.Hur,J.Park, W.Woo, K.Lim, C.Lee, H.Kim and J.Lasker, "A wideband analog multi-resolution spectrum sensing (MRSS) techniques for cognitive radio (CR) systems," in Island of KOS, Greece, 2006.

[22] G.Ganesan and Y.Li, "Agility improvement through cooperative diversity in cognitive radio," in IEEE Global telecommunication conference (GLOBECOM '05), Missouri, USA, Dec. 2005.

[23] S.D.Jones, E.Jung, X.Liu, N.Merheb and I.J.Wang, "Characterization of spectrum activities in the US public safety band for opportunistic spectrum access," in International symposium on new frontiers in dynamic spectrum access networking (DySPAN '07), Dublin, Ireland, APR. 2007.

[24] C.Cordeiro, K.Challapali, D.Birnu and S.Shankar, " IEEE 802.22; the first wiorldwide wireless standard based on cognitive radio," in IEEE international symposium on new frontiers in dynamic spectrum access network (DySPAN'05), Baltimore, MD, USA, Nov. 2005.

[25] A.Ghasemi and E.S.Sousa, "Capacity of fading channel under spectrumsharing constraints," in IEEE international conference on communications, Istanbu, Turkey, 2006.

[26] P.Wang, L.Xiao, S.Zhou and J.Wang, "Optimization of detection time for channel efficiency in cognituve radio systems," in IEEE wireless communication and networking conference, Hong Kong, 2005.

[27] N.Khambekar, L.Dong and V.Chaudhery, "Utilizing OFDM guard interval for spectrum sensing," in IEEE wireless communication and networking conference, Hong Kong, Mar. 2007.

[28] W.Hu, D.Wikomm, M.Abusubaih, J.Gross, G.Vlantis, M.Gerla and A.Wolisz, "Dynamic frequency hopping communities for efficient IEEE
802.22 operation," IEEE communication magazine, vol. 45, no. 5, pp. 80 - 87, May 2007.

[29] E.Visotsky, S.Kuffner and R.Petterson, "On collaborative detection on TV transmission in support of dynamic spectrum sharing," in Proceedings of IEEE International symposium of New Frontiers in Dynamic spectrum access network, Baltimore, Nov 2005.

[30] T.Weiss, J.Hillenbrand and F.Jondral, "A diversity approach for the detection of Idle spectral resource in spectrum pooling systems," in Proceedings of the 48th International scientific colloqium, IImenau, Germany, 2003.

[31] Z.Chair and P.K.Varshney, "Optimal data fusion on multiple sensor detection system," IEEE transaction on Aerospace Electronics system, vol. 22, no. 1, p. $98101,1986$.

[32] M.Gandetto, A.F.Catto, C.S.Regazzoni and M.Musso, "Distributed cooperative mode identification for cognitive radio application," in Proceedings of international radio science union (URSI), New Delhi, India, 2005.

[33] M.Gandetto, A.F.Cattoni, C.S.Regazzoni, "Distributted approach to mode identification and spectrum monitoring for cognitive radio," in Proceedings of SDR forum for technical conference, Orange County, California, USA, Nov. 2005.

[34] A.F.Cattoni, I.Minetti, M.Gandetto, R.Niu, P.K.Varshney and C.S.Regazzoni, "A Spectrum sensing algorithm based on distributed cognitive model," in Proceeding of SDR forum for technical conference, Orlando, Florida, USA, Nov.2006.

[35] M.Gandetto andf C.S.Regazzoni, "Spectrum Sensing: A distributed approach for cognitive terminals," IEEE Journal on selected areas of communication, vol. 25, no. 3, pp. 546 - 557, 2007.

[36] P.Pawelczar, G.J.Janssen and R.V.Prasad, "Performance measure of dynamic spectrum access networks," in Proceedings of IEEE Global telecommunication conference (Globecom), San Francisco, California USA, Nov.2006.

[37] R.Chen and J.M.Park, "Ensuring trustworthy spectrum sensing in cognitive radio network," in Proceedings of IEEE workshop on networking technologies for software defined radio networks (held in conjunction with IEEE SECON 2006), 2006.

[38] E.Orumwense, O.Olutayo, S.Mneney, "Impact of primary user emulation attack on cognitive radio network," International journal on communication antenna on propation, vol. 4, no. 1, pp. 19 - 26, 2014.

[39] C.N.Mathur and K.P.Subbalakshmi, "Digital signatures for centralized DSA network," in First IEEE workshop on cognitive radio networks, Las Vegas, Nevada, USA, Jan 2007.

[40] S.M.Kay, Fundamentals of statistical signal processing : Detection Theory, Upper Saddle River, NJ: Prentice Hall, 1998.

[41] H.V.Poor, An Introduction to signal detection and estimation, Berlin: Springer, 1988.

[42] H.L. Van-Tress, Detection, Estimation and Modulation Theory, New York: John Wiley \& Sons, 2001.

[43] Y.Zheng, Y.C.Liang, A.T.Hoang and R.Zheng, "A Review on spectrum sensing for cognitive radio:challenges and solutions," EURASIP Journal and advances in signal processing, 2010.

[44] E.Fishler, A.Haimovich, R.Blum, D.Chizhik,L.Cimini and R.Valenzuela, "MIMO Radar: an idea whose time has come," in Proceedings of the IEEE National rada conference, Philadephia, USA, Apr.2004.

[45] A.Sheiki and Zamani, "Coherent detection for MIMO Radars," in Proceedings of IEEE National Radar Conference, Apr. 2007.

[46] P.Stoica, J.Li and Y.Xie, On Probing signal processes advances in wireless and mobile communications, vol. 1, Prentice Hall, 2001.

[47] M.Subheder and G.Birajdar, "Spectrum sensing techniques in cognitive radio: A Survey," International Journal of Next-Generation Networks (IJNGN), vol. 3, no. 2, Jun 2011.

[48] J.G.Proakis, Digital Communication, 4th ed., McGraw Hill, 2001.

[49] R.F.Ustok, Spectrum sensing techniques for cognitive radio systems with multiple antennas, 2010.

[50] S.Shankar, C.Cordeiro and K.Challapali, "Spectrum agile radio: utilization and sensing architectures," in IEEE international symposium 
on new frontiers in dynamic spectrum access network (DySPAN'05), Baltimore, Maryland, USA, Nov. 2005.

[51] D.Cabric, S.Mishra and Brodersen, "Implementation issues in spectrum sensing for cognitive radios," in Proceedings of Asilomar conference on signal systems and computers, California, USA, No. 2004.

[52] K.Maeda, A.Benjaebbour, T.Asai, T.Furuno and T.Onya, "Recongnition array OFDM-Based systems utilizing cyclostationarity inducing transmission," in Proceedings of IEEE international symposium on New Frontiers in Dynamic spectrum access networks, Dublin, Ireland, Apr.2007.

[53] W.A.Gardner, A.Napolitanob and L.Paurac, "Cyclostainoarity:Half a ccentury of research," Elsevier signal processing, vol. 86, pp. 639 - 697, 2006.

[54] Y.Tengyiz and G.Chi, "Performance of Cyclostationary feature based spectrum sensing methods in multiple antenna cognitive systems," in Wireless communication and networking conference (WCNC), 2009.

[55] M.Hoyhtya, A.Hekkala, M.Katz and A.Mammela, "Spectrum Awareness: Techniques and challenges for active spectrum sensing," in Cognitive wireless networks, 2007.

[56] T.Yucek, Channel, Spectrum and wave form Awareness in OFDMBased Cognitive Radio, 2007.

[57] N.C.Beaulieu and Y.Chen, "Improved energy detectors for cognitive radios with randomly arriving and departing primaryusers," IEEE signal processing letters, vol. 17, no. 10, pp. 870 - 877, 2010.

[58] L.Tang, Y.Chen, E.L.Hines and M.S.Alouini, "Effect of primary user traffic on sensing-throughput tradeoff for cognitive radios," IEEE Transaction on wireless communication , vol. 8, no. 4, pp. 1063 -1068, 2011.

[59] H.Tang, "Some physical layer issues of wiiderband cognitive radio system," in Proceeding of IEEE international sysmposium of New Frontiers in Dynamic Spectrum access networks, Baltimore, Maryland, USA, Nov.2005.

[60] Y.Zeng, C.L.Koh and Y.C.Liang, "Maximum Eigenvalue Detection:Theory and Application," in IEEE International conference on communication, 2007.

[61] A.M.Tulino and S.Verdu, Random Matrix Theory and, Now Publisher Inc, 2004.

[62] Y.Zeng and Y.C.Liang, "Eigenvalue- Based spectrum algorithm for cognitve radio," IEEE Transaction on communication, vol. 57, no. 6, 2009.

[63] M.E.Yildizy, T.C.Aysaly and K.E.Barner, "In network cooperative spectrum sensing," in Proceedings of EURASIP European signal processing conference, Glasgow, UK, 2009.

[64] X.Jing, D.Raychandhuri, "CSCE etiquette protocol," in IEEE DySPAN, 2005.

[65] C.Sun, W.Zhang and K.B.Letaief, "Cooperative spectrum sensing for cognitive radio under bandwidth constraints," in In proceedings of IEEE communication and networking conference, Hong Kong, Mar 2001.

[66] Z.Li, F.R.Yu and M.Huang, "A distributed consensus based cooperative spectrum sensing scheme in cognitive radio," IEEE transaction on vehicular technology, vol. 59, no. 1, pp. 383 - 393, 2010.

[67] W. K.B.Letaief, "cooperative communication for cognitive radio networks," proceeding of IEEE, vol. 97, no. 5, pp. 878 - 893, 2009.

[68] [68]J.Ma, G. Yeli and B.H. Juang, "Signal processing in cognitive radio," proceeding of IEEE, vol. 97, no. 5, May 2009.

[69] [69]Y.Zeng , C.L.Koh and Y.C.Liang, "Maximum eigenvalue detection: theory and application," in IEEE International conference on communication, 2007. 